\title{
8-氨基喹啉类铜离子螯合剂的合成及螯合选择性研究
}

\author{
黄达涯 $^{a}$ 李佑智 ${ }^{a}$ 刘 艳*,a Bernard Meunier ${ }^{*, a, b}$ \\ $\left({ }^{a}\right.$ 广东工业大学轻工化工学院 广州 510006) \\ $\left({ }^{b}\right.$ Laboratoire de Chimie de Coordination du CNRS, Centre National de la Recherche Scientifique, 205 route de Narbonne,
}

BP 44099, 31077 Toulouse cedex 4, France)

\begin{abstract}
摘要 阿尔茨海默(AD)脑内氧化还原活性金属离子稳态失衡, 尤其是铜离子的稳态失衡, 是引起氧化应激, 从而导致 $\mathrm{AD}$ 脑内氧化损伤的重要原因. 为了开发选择性的铜离子螯合剂, 以开发抑制 $\mathrm{AD}$ 氧化应激先导化合物，以 2-甲基-8-硝 基喹啉为原料, 经过硝基还原、 $\mathrm{C} 2$ 位氧化(官能团化)、还原胺化、加成等反应合成了具有四配位鳌合作用的喹啉衍生 物. 通过调节侧链的长度及配位原子的位置, 对化合物的络合能力、对 $\mathrm{Cu}(\mathrm{II})$ 和 $\mathrm{Zn}(\mathrm{II})$ 的选择性进行了研究. 其中, TDMQ29 对铜离子有特异选择性, $\log K_{\text {app }}\left[\mathrm{Cu}^{\mathrm{II}}-\mathrm{TDMQ} 29\right]$ 为 15.7, 而 $\log K_{\text {app }}[\mathrm{Zn}$-TDMQ29] 为 5.8, 对铜离子的络合常数 比对锌离子的络合常数高出 10 个数量级.
\end{abstract}

关键词＼cjkstart阿尔茨海默症; 8-氨基喹啉; 铜离子螯合; 选择性

\section{Synthesis and Chelation Selectivity Evaluation of 8-Aminoquinoline Derivatives as Copper Chelator}

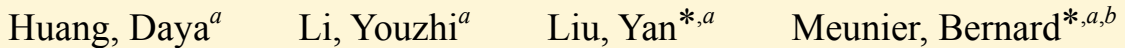 \\ $\left({ }^{a}\right.$ School of Chemical Engineering and Light Industry, Guangdong University of Technology, Guangzhou 510006) \\ $\left({ }^{b}\right.$ Laboratoire de Chimie de Coordination du CNRS, Centre National de la Recherche Scientifique, \\ 205 Route de Narbonne, BP 44099, 31077 Toulouse Cedex 4, France)
}

\begin{abstract}
The non-controlled redox-active metal ions, especially copper, in the brain of patients with Alzheimer disease (AD) should be considered at the origin of the intense oxidative damage in AD brain. In this paper, we designed and synthesized new compounds based on 8-aminoquinoline motif with a lateral chain at the $\mathrm{C} 2$ position of the aromatic ring. The affinities for $\mathrm{Cu}$ (II) and $\mathrm{Zn}$ (II) of these ligands are reported in the present article. Among these chelators, TDMQ29 is specific for copper chelation with $\log K_{\text {app }}\left[\mathrm{Cu}^{\text {II }}\right.$-TDMQ29] to be 15.7 and $\log K_{\text {app }}\left[\mathrm{Zn}^{\mathrm{II}}-\mathrm{TDMQ29]}\right.$ to be 5.8. Such metal ligands can be considered as potential ligands, which are able to regulate the homeostasis of copper in brains.

Keywords Alzheimer disease (AD); 8-aminoquinoline; copper chelation; selectivity
\end{abstract}

阿尔茨海默症(Alzheimer’s disease, AD)是常发于老 年人群的一种慢性神经退行性疾病, 其临床表现为记忆 力衰退、判断力和理解力下降等症状. 据阿尔茨海默协 会统计, 全世界目前已有超过 2400 万 $\mathrm{AD}$ 患者, 预计未 来 20 年患者数量将翻一番 ${ }^{[1,2]}$. $\mathrm{AD}$ 严重影响患者的工作 能力和生活质量, 也为家庭和社会带来沉重的负担. 而 我国，正步入老龄化社会，据统计 1990 年我国有 $\mathrm{AD}$ 患
者 190 万，至 2010 年已增加至 560 万人 ${ }^{[2 ~ 4]}$. 日益增加 的 $\mathrm{AD}$ 患者正给我国的医疗和经济带来巨大压力 ${ }^{[2 \sim 4]}$. 因此, 研究和开发 $\mathrm{AD}$ 治疗药物具有重要的临床意义和 社会价值.

目前, 用于 $\mathrm{AD}$ 治疗的临床药物主要包括几个乙酰 胆碱酯酶抑制剂以及谷氨酸亚型 $N$-甲基- $D$-天冬氨酸 (NMDA)受体拮抗剂美金刚(Memantine $)^{[5,6]}$. 虽然, 这些

\footnotetext{
* Corresponding authors. E-mail: yanliu@gdut.edu.cn; bmeunier@lcc-toulouse.fr

Received May 4, 2018; revised July 31, 2018; published online September 5, 2018.

Project supported by the National Natural Science Foundation of China (No. 21502023), the Open Project of Guangdong Provincial Key Laboratory of New Drug Screening (No. GDKLNDS-2018OF004), the Program for Innovative Research Teams and Leading Talents Introduction of Guangdong Province (No. 2050205), and the Guangdong University of Technology (No. 220418037).

国家自然科学基金(No.21502023)、广东省新药笁选重点实验室开放课题(No. GDKLNDS-2018OF004)、广东省领军人才(No. 2050205)、广东工业大学 (No. 220418037)资助项目.
} 
药物在一定程度上可以相对减轻 $\mathrm{AD}$ 的症状, 但不能阻 止病情的进展, 疗效非常有限, 且效率/成本比备受质 疑. 自 2003 年食品药品监督管理局(FDA)批准美金刚以 来, 过去 15 年所有 $\mathrm{AD}$ 药物的研究都在不同临床阶段宣 告失败, 失败率 $99.6 \%{ }^{[7]}$. 因此, 开发有效的治疗药物, 在 $\mathrm{AD}$ 发病早期从根本上改善疾病的状态或终止疾病的 进程是当今国际医药领域的热点, 也是亟待突破的领域 之一.

$\mathrm{AD}$ 的病理特征是淀粉样蛋白沉淀以及高度磷酸化 tau 蛋白的异常沉积. 然而, $\mathrm{AD}$ 是一种复杂的多因素疾 病, 广泛的研究证实, $\mathrm{AD}$ 与以下因素密切相关: (1) tau 蛋白、(2)淀粉样蛋白聚集、(3)金属离子紊乱等. 研究表 明, 这三个因素是密切相关的 ${ }^{[8 ~ 10]}$. 例如, 由淀粉样蛋 白前体产生过量的淀粉样蛋白, 是形成 $\mathrm{AD}$ 的重要因素, 然而, 淀粉样蛋白聚集本身并不是引起疾病的充分条 件.

$\mathrm{AD}$ 患者脑内金属的稳态失衡是引起氧化应激从而 引起神经元死亡的重要原因. 尸检分析表明, $\mathrm{AD}$ 患者脑 内老年斑块中心及周围金属离子的水平明显高于正常 生理水平, 铜、铁和锌的过量积累分别是正常大脑的 5.7、2.9 和 2.8 倍. 尤其是铜离子的含量: 正常生理条件 下, 脑内 $\mathrm{Cu}$ 离子的浓度为 $70 \mu \mathrm{mol} \cdot \mathrm{L}^{-1}$; 而 $\mathrm{AD}$ 患者脑 内的老年斑块中沉积的 $\mathrm{Cu}$ 离子的浓度为 $400 \mu \mathrm{mol} \cdot \mathrm{L}^{-1}$, 竟高达近 6 倍 $^{[9-11]}$. 脑内铜离子异常会导致 $\mathrm{Cu}$-淀粉样 蛋白 $(\mathrm{Cu}-\mathrm{A} \beta)$ 复合物的产生, 该复合物在内源性还原物 质的存在性会诱导产生活性氧蔟(reactive oxygen species, ROS), 介导脑内氧化应激, 从而导致神经元的死 $亡^{[12 \sim 14]}$. 因此, 开发有效的铜离子螯合剂, 从 $\mathrm{Cu}-\mathrm{A} \beta$ 复 合物中提取铜离子，抑制 $\mathrm{Cu}-\mathrm{A} \beta$ 复合物介导的氧化应 激, 对阻止 $\mathrm{AD}$ 脑内氧化损伤、逆转 $\mathrm{AD}$ 记忆损伤具有 重要作用.

Bush 等 ${ }^{[15 \sim 17]}$ 开发了铜、锌离子螯合剂氯碘羟喹 (CQ, Clioquinol), 及 CQ 衍生物 PBT2(结构如图 1 所示), 二者均为二齿螯合配合物. 研究发现, CQ 和 PBT2 与 $\mathrm{Cu}-\mathrm{A} \beta$ 复合物形成新的 $L-\mathrm{Cu}-\mathrm{A} \beta$ 复合物, 而不能从 $\mathrm{Cu}-\mathrm{A} \beta$ 复合物中夺取铜离子 ${ }^{[18]}$. 另一方面, CQ 和 PBT2 对铜离子的选择性较差, 在络合铜离子的同时导致锌离 子的紊乱, 从而导致严重的毒性 ${ }^{[19]}$. 因此, 基于抗 $\mathrm{AD}$ 氧化应激思路设计理想的铜离子螯合剂, 以恢复脑内铜 离子的稳态平衡, 关键在于螯合剂应具有良好的铜离子 选择性，既螯合铜离子的同时不破坏其它金属离子的平 衡, 尤其是锌离子的稳态平衡.

为了开发选择性的铜离子调节剂, 本论文以 8-氨基 喹啉为母核, 通过在 $\mathrm{C} 2$ 位引入柔性侧链及配位原子, 设计合成了具有四配位螯合作用的喹啉衍生物(Tetra<smiles>O=C(O)c1c(I)cc(Cl)c2cccnc12</smiles><smiles>CN(C)Cc1ccc2c(Cl)cc(Cl)c(O)c2n1</smiles>

图 $1 \mathrm{CQ}$ 和 $\mathrm{PBT} 2$ 的结构

Figure 1 Structure of CQ and PBT2

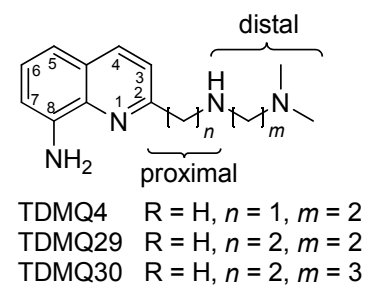

图 2 四配位喹啉衍生物 TDMQ 的结构

Figure 2 Structure of synthesized TDMQ ligands

Dentate Mono Quinolines, TDMQ, 如图 2 所示), 并通过 调节侧链的长度及配位原子的位置对衍生物的络合能 力, 以及衍生物对铜离子、锌离子的选择性进行了研究.

\section{1 结果与讨论}

\section{1 目标化合物的合成}

化合物 TDMQ4 以 2-甲基-8-硝基喹啉为起始原料 经五步反应获得. 合成路线如 Scheme 1 所示, 以 2-甲 基-8-硝基喹啉(1)为原料, 在乙醇和冰醋酸中采用铁粉 还原得到 2-甲基- 8 -氨基喹啉(2), 收率为 $88 \%$, 对 C- 8 氨 基进行乙酰基保护后, 用 $\mathrm{Se}_{2} \mathrm{O}$ 将 $\mathrm{C}-2$ 甲基氧化为醛基得 化合物 4, 收率为 $65 \%$, 化合物 $\mathbf{4}$ 与 $N, N$-二甲基乙二胺 进行亲核加成反应, 经 $\mathrm{NaBH}(\mathrm{OAc})_{3}$ 还原, 对 $\mathrm{C}-8$ 氨基 去保护, 得化合物 $\mathbf{5}$, 收率可达 $93 \%$, 化合物 $\mathbf{5}$ 在盐酸乙 醚溶液中可定量地生成盐酸盐, 得到目标物 TDMQ4.

为了研究侧链的长度及配位原子的位置对衍生物 的络合能力的影响, 我们设计合成了化合物 TDMQ29 和 TDMQ30. 合成路线如 Scheme 2 所示, 以 2-甲基-8硝基喹啉(1)为原料, 在 $N, N$-二甲基乙酰胺(DMA)中以 $\mathrm{FeCl}_{3}-\mathrm{K}_{2} \mathrm{~S}_{2} \mathrm{O}_{8}$ 为氧化体系, 在 $110{ }^{\circ} \mathrm{C}$ 搅拌反应 $30 \mathrm{~min}$, 将 $\mathrm{C}-2$ 位芳甲基进行氧化官能化为 2-乙烯基-8-硝基喹 啉(6), 收率为 51\%. 化合物 $\mathbf{6}$ 与氨基化合物进行亲核加 成反应, 在 $\mathrm{C}-2$ 位引入含 $\mathrm{N}$ 原子的柔性脂肪链, 得到化 合物 $7 \mathbf{a}$ 和 $7 \mathbf{b}$, 收率分别为 $46 \%$ 和 $89 \%$. 化合物 $7 \mathbf{a}$ 和 $7 \mathbf{b}$ 的 C-8 位硝基经 $\mathrm{SnCl}_{2}$ 还原后, 得到化合物 $8 \mathbf{a}$ 和 $8 \mathbf{b}$, 产 率分别为 $61 \%$ 和 $73 \%$. 化合物 $8 \mathrm{a}$ 和 $8 \mathrm{~b}$ 在盐酸乙醚溶液 中成盐酸盐, 分别得到目标物 TDMQ29 和 TDMQ30.

\section{2 化合物与 $\mathrm{Cu}(\mathrm{II})$ 和 $\mathrm{Zn}(\mathrm{II})$ 络合的计量比}

参照文献方法 ${ }^{[3,5,20]}$, 化合物与 $\mathrm{Cu}(\mathrm{II})$ 和 $\mathrm{Zn}(\mathrm{II})$ 络合 


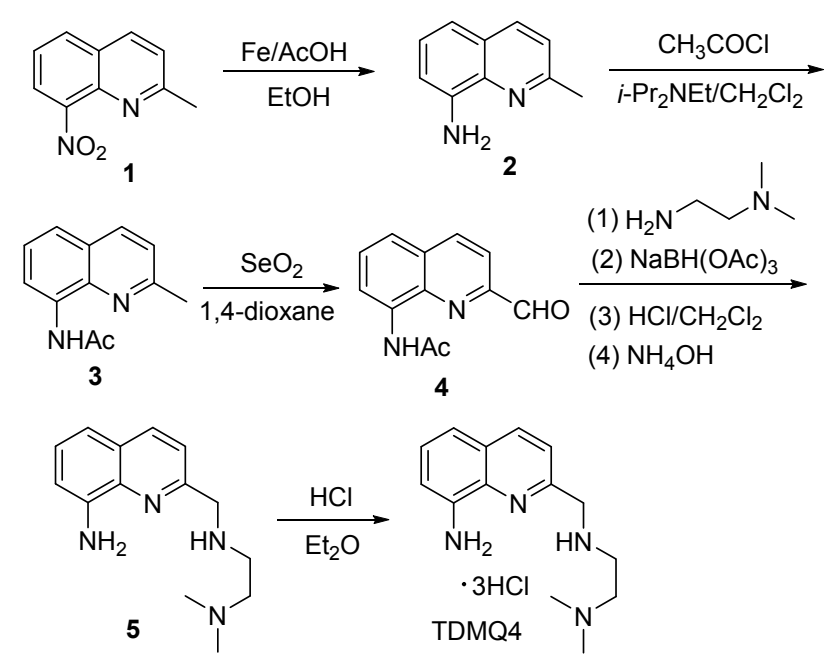

图式 1 化合物 TDMQ4 的合成

Scheme 1 Synthetic route for TDMQ4
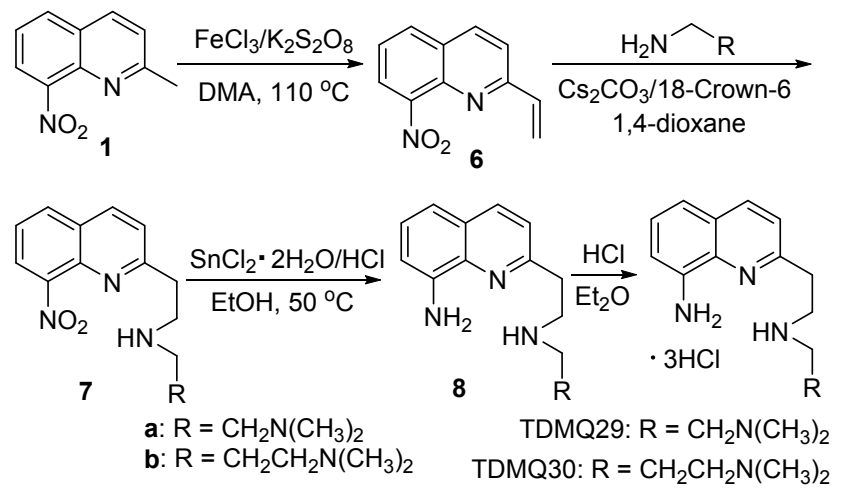

图式 2 化合物 TDMQ29 和 TDMQ30 的合成

Scheme 2 Synthetic route for TDMQ29 and TDMQ30

的计量比采用紫外滴定法测试. 以化合物 TDMQ29 为 例, 实验在 $\mathrm{Tris} \cdot \mathrm{HCl}\left(20 \mathrm{mmol} \cdot \mathrm{L}^{-1}\right) / \mathrm{NaCl}(150 \mathrm{mmol} \cdot$ $\mathrm{L}^{-1}$ ) 缓冲溶液 $(\mathrm{pH}$ 7.4) 中进行, 向化合物 TDMQ29 (15 $\left.\mu \mathrm{mol} \cdot \mathrm{L}^{-1}\right)$ 中分别加入不同当量的铜离子 $(0.3 、 0.5 、 0.8$ 、 $1.0 、 1.1 、 1.2 、 1.5 、 2.0$ equiv.), 化合物与铜离子发生快 速络合反应, 化合物 TDMQ29 与铜离子络合的紫外-可 见光谱吸收及吸收变化如图 3 所示. 化合物 TDMQ 29 在 250 及 $350 \mathrm{~nm}$ 处有特征吸收, 随着铜离子加入量的增 加, TDMQ29 在 250 及 $350 \mathrm{~nm}$ 处的吸光度呈线性减弱, 说明 TDMQ29 的量在逐渐减少. 当加入的铜离子为 1.0 当量时, TDMQ29 与 $\mathrm{Cu}(\mathrm{II})$ 的络合达到平衡, 而加入的 铜离子的物质的量大于 1.0 equiv. 时, 在 250 及 $350 \mathrm{~nm}$ 处的吸光度不再变化. 另一方面, $\mathrm{Cu}^{\mathrm{II}}-\mathrm{TDMQ} 29$ 络合物 的特征吸收波长为 $234 \mathrm{~nm}$, 随着铜离子的加入, $234 \mathrm{~nm}$ 处的吸光度逐渐增强, 说明随着铜离子的增加 $\mathrm{Cu}^{\mathrm{II}}-$ TDMQ29 络合物的浓度不断增加. 当加入的铜离子的 物质的量大于 1.0 equiv.时, $234 \mathrm{~nm}$ 处的吸光度不再变 化. 由此得知化合物 TDMQ29 与铜离子的络合比为 1 : 1. 采用同样的实验方法测得 TDMQ4, TDMQ30 与铜离
子的络合比为也分别为 $1: 1$, 其紫外-可见光吸收谱图 见辅助材料.

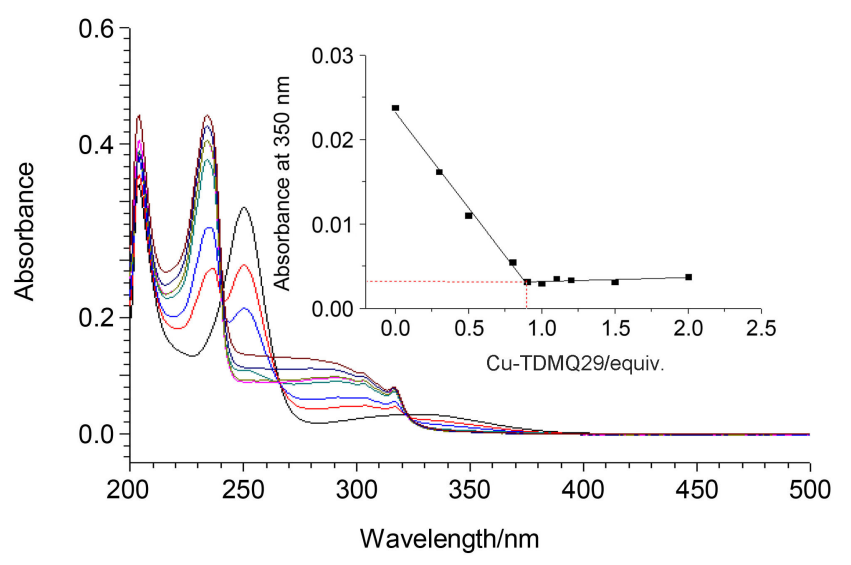

图 3 紫外滴定方法测试化合物 TDMQ29 与铜离子的计量比 Figure 3 Determination of the stoichiometry by UV-Vis titration of TDMQ29 with $\mathrm{Cu}(\mathrm{II})$

以化合物 TDMQ29 为例, 采用紫外滴定法测试螯 合剂与 $\mathrm{Zn}(\mathrm{II})$ 络合的化学计量比. 实验在 Tris $\cdot \mathrm{HCl}(20$ $\left.\mathrm{mmol} \cdot \mathrm{L}^{-1}\right) / \mathrm{NaCl}\left(150 \mathrm{mmol} \cdot \mathrm{L}^{-1}\right)$ 缓冲溶液 $(\mathrm{pH}$ 7.4)中进 行，向化合物 TDMQ29 $\left(15 \mu \mathrm{mol} \cdot \mathrm{L}^{-1}\right)$ 中分别加入不同 量的锌离子，直至化合物与锌离子完全络合. 化合物 TDMQ29 与锌离子络合的紫外-可见光吸收谱及吸收变 化如图 4 所示. 化合物 TDMQ29 在 $250 \mathrm{~nm}$ 处有最大吸 收, 随着锌离子加入量的增加, 该处吸收逐渐减弱, 与 此同时, $237 \mathrm{~nm}$ 处是 $\mathrm{Zn}{ }^{\mathrm{II}}-\mathrm{TDMQ} 29$ 络合物的特征吸收波 长, 随着锌离子的加入, 该处的吸光度逐渐增强, 由此 可判断 $\mathrm{Zn}^{\mathrm{II}}-\mathrm{TDMQ} 29$ 络合物的生成随着锌离子浓度的 增加而增加. 当加入的锌离子的物质的量大于 30 equiv. 时, 在 $250 \mathrm{~nm}$ 处可观察到吸光度不再变化, 在此吸光度 下根据锌离子的物质的量与吸光度的关系绘制出曲线 图, 化合物 TDMQ29 与锌离子的络合比为 $1: 30$. 同样 的方法测得, TDMQ4 与锌离子的络合比小于 $1: 30$, TDMQ30 与锌离子的络合比小于 $1: 300$. 其紫外-可见 光吸收谱图及其吸收变化见辅助材料. 如图 4 所示, 根 据吸光度与加入的锌离子的物质的量的关系作图得出 当锌离子与化合物达到 $1 / 2$ 络合时, 所需加入的锌离子 量为 0.57 equiv., 根据平衡常数的计算方法得出化合物 TDMQ29 与锌离子的络合比为 5.8 (具体计算方法见实验 部分). TDMQ4, TDMQ29, TDMQ30 与锌离子的络合常 数见表 1 .

\section{3 化合物对 $\mathrm{Cu}(\mathrm{II})$ 和 $\mathrm{Zn}(\mathrm{II})$ 的络合能力及络合选择性}

化合物与铜离子的络合常数通过紫外-可见光谱法 测定 ${ }^{[3.5 .20]}$, 使用乙二胺四乙酸(EDTA)、乙二醇双(2-氨基 乙基醚)四乙酸(EGTA)、氨基三乙酸(NTA)、乙二胺 $(E D A)$ 作为竞争性螯合剂. 化合物与铜离子等物质的量 


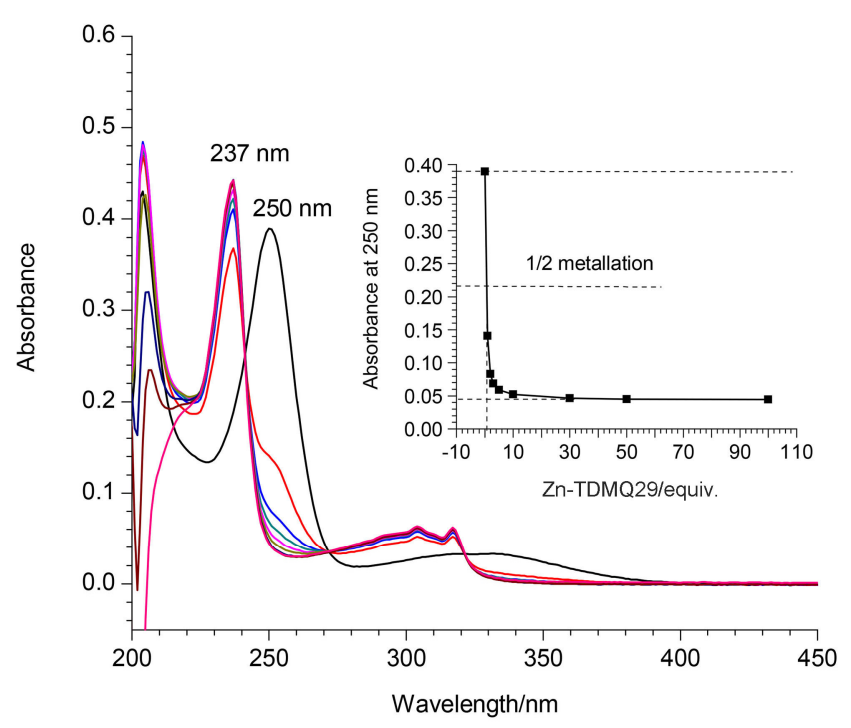

图 4 紫外滴定方法测试化合物 TDMQ29 与锌离子的计量比 Figure 4 Determination of the stoichiometry by UV-Vis titration of TDMQ29 with Zn(II)

表 1 化合物与 $\mathrm{Cu}(\mathrm{II})$ 和 $\mathrm{Zn}(\mathrm{II})$ 的络合常数及络合选择性

Table 1 Affinity constants of TDMQ ligands for $\mathrm{Cu}(\mathrm{II})$ and $\mathrm{Zn}(\mathrm{II})$, and selectivity for $\mathrm{Cu}(\mathrm{II})$ with respect to $\mathrm{Zn}$ (II)

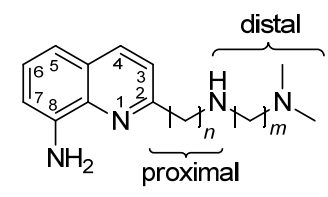

\begin{tabular}{cccccc}
\hline \multirow{2}{*}{ Compd. } & \multirow{2}{*}{$n$} & $m$ & \multicolumn{2}{c}{$\log K_{\mathrm{app}}\left[\mathrm{M}^{\mathrm{II}}-\mathrm{L}\right]^{a}$} & \multirow{2}{*}{${ }^{\mathrm{II}} \mathrm{Zn}^{\mathrm{II}} b$} \\
\cline { 4 - 5 } & & & $\mathrm{M}=\mathrm{Cu}^{\mathrm{II}}$ & $\mathrm{M}=\mathrm{Zn}^{\mathrm{II}}$ & \\
\hline TDMQ4 & 1 & 2 & 11.7 & 5.3 & 6.4 \\
TDMQ29 & 2 & 2 & 15.7 & 5.8 & 9.9 \\
TDMQ30 & 2 & 3 & 10.1 & $<3$ & $>7.1$ \\
\hline
\end{tabular}

${ }^{a}$ Affinity constants of TDMQ ligands were determined by using UV-Vis spectrometry. ${ }^{b}$ Selectivity of TDMQ ligands for $\mathrm{Cu}(\mathrm{II})$ with respect to $\mathrm{Zn}$ (II): $\log K_{\text {app }}\left[\mathrm{Cu}^{\mathrm{II}}-\mathrm{L}\right]-\log K_{\text {app }}\left[\mathrm{Zn}^{\mathrm{II}}-\mathrm{L}\right]$

比络合后产生明显的蓝移, 加入等物质的量的竞争性螯 合剂后, 部分铜离子被竞争性螯合剂夺走. 根据达到平 衡时铜离子与化合物络合的百分比来计算化合物与铜 离子的络合常数. 如图 5 所示, 当 $\mathrm{TDMQ} 29 / \mathrm{Cu} / \mathrm{EDTA}=$ $1 / 1 / 1$ 达到平衡时, 在 $350 \mathrm{~nm}$ 处, 计算可得 TDMQ29 与 铜离子络合的百分比 $x$ 为 $53 \%$. 计算可得 TDMQ29 与铜 离子的络合常数为 15.7 .

采用相同的实验方法, TDMQ4 与铜离子的络合常 数为 $11.7, \mathrm{TDMQ} 30$ 与铜离子的络合常数为 10.1(表 1). 对比 TDMQ4, TDMQ29 与 TDMQ30 的化学结构, TDMQ29 与 TDMQ4 的 $n$ 相差 1 , 即侧链上临近喹啉环 的 $\mathrm{N}$ 原子与喹啉母核之间的距离相差一个亚甲基，而 TDMQ29 与 TDMQ30 的 $m$ 相差 1, 即侧链上参与金属 螯合的两个 $\mathrm{N}$ 原子之间的距离相差一个亚甲基. 构效关 系表明最佳侧链长度应为 $n=2, m=2$. 而改变化合物侧 链的长度及改变侧链上参与螯合的 $\mathrm{N}$ 原子之间的距离,
对化合物与铜离子的络合能力影响较大.

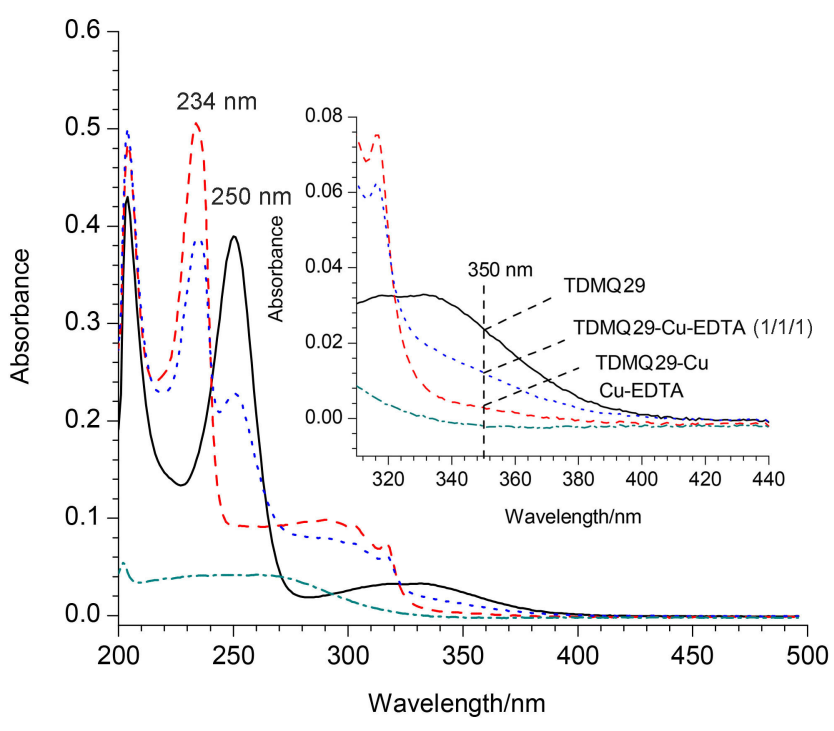

图 5 紫外-可见光谱法测定 TDMQ29 与铜离子的络合常数

Figure 5 Determination of $\log K_{\text {app }}$ of $\mathrm{Cu}^{\text {II }}-\mathrm{TDMQ} 29$ by using UV-visible spectrometry

合成的化合物与锌离子的络合能力较弱, TDMQ29 与锌离子作用时, 根据吸光度与加入的锌离子的物质的 量的关系作图, 得出当 $1 / 2$ 的 TDMQ29 被络合生成 $\mathrm{Zn}{ }^{\mathrm{II}}$ TDMQ29 络合物时, 需加入的锌离子 0.57 equiv. (如图 4 所示), 根据文献方法计算得出化合物 TDMQ29 与锌离 子的络合比为 5.8 (具体计算方法见实验部分). TDMQ4 和 TDMQ 30 与锌离子的络合常数分别为 5.3 及 $<3.0$, 见 表 1 .

由表 1 可知, TDMQ 系列化合物与铜离子的络合常 数为 $10 \sim 16$, 对锌离子的络合常数小于 6 , 相差了 6 10 个数量级, 对铜离子有选择性螯合作用. 其中, TDMQ29 与铜离子的络合常数为 15.7 , 而与锌离子的络 合常数为 5.8 , 相差 10 个数量级, 表明该螯合剂对铜离 子有较强的选择性. TDMQ29 可作为先导化合物, 进行 进一步结构修饰和深入的构效关系研究.

\section{2 结论}

本实验设计合成了 3 个四配位的铜离子螯合剂并利 用核磁共振、高分辨质谱和元素分析对目标化合物进行 结构确认. 采用紫外滴定方法, 对化合物与 $\mathrm{Cu}(\mathrm{II})$ 和 $\mathrm{Zn}(\mathrm{II})$ 的络合计量比进行测定, 发现该系列化合物与 $\mathrm{Cu}(\mathrm{II})$ 的络合比为 $1: 1$, 作用较强, 而与 $\mathrm{Zn}(\mathrm{II})$ 的络合计 量比 $<1: 30$, 络合能力较弱. 进一步采用紫外-可见光 谱法对化合物与 $\mathrm{Cu}(\mathrm{II})$ 和 $\mathrm{Zn}(\mathrm{II})$ 的络合常数进行了测定, 并对 TDMQ 系列化合物进行了构效关系的探究. 实验 结果显示, TDMQ 系列化合物对铜离子都有较好的选择 性, 与铜离子的络合常数比对锌离子的络合常数相差 
6 10 个数量, 其中 TDMQ29 和铜离子的络合常数为 15.7 , 而对锌离子的络合常数为 5.8 , 两者相差约 10 个 数量级, 表明 TDMQ29 对铜离子有较强的选择性螯合 作用, 可作为先导化合物进行深入研究. 对比 TDMQ4, TDMQ29 与 TDMQ30 的化学结构特征, 其主要区别在 于 $\mathrm{C} 2$ 位柔性链的长短, 以及侧链上鳌合因子之间的距 离不同, 构效关系表明改变化合物侧链的长度及改变侧 链上参与螯合的 $\mathrm{N}$ 原子之间的距离, 对化合物与铜离子 的络合能力影响较大, 最佳侧链长度为 $n=2, m=2$.

\section{3 实验部分}

\section{1 仪器与试剂}

${ }^{1} \mathrm{H}$ NMR 和 ${ }^{13} \mathrm{C}$ NMR 使用布鲁克 $400 \mathrm{MHz}$ 超导核 磁共振仪测定, 样品以氛代氯仿 $\left(\mathrm{CDCl}_{3}\right)$ 或重水 $\left(\mathrm{D}_{2} \mathrm{O}\right)$ 为 溶剂, 四甲基硅烷(TMS)为内标; 紫外滴定使用 Thermo Evolution 300 测定; 质谱使用 ThermoFischer (DCI) 或者 GCT Premier (DCI, CH4, HRMS)测定; 元素分析使用 varioELcube 测定. 实验用的试剂和原料均为分析纯或 化学纯.

\section{2 实验方法}

\subsection{1 化合物 TDMQ4 的合成}

常温下，向 2-甲基-8-硝基喹啉 $(850 \mathrm{mg}, 4.52 \mathrm{mmol}$ ) 的乙醇溶液 $(9 \mathrm{~mL})$ 中加入还原铁粉 $(757 \mathrm{mg}, 13.55$ $\mathrm{mmol}$ )和冰醋酸 $(3 \mathrm{~mL})$, 回流反应 $4 \mathrm{~h}$ 后, 将反应冷却至 室温, 向体系中缓慢加入饱和 $\mathrm{NaHCO}_{3}$ 水溶液, 用二氯 甲烷萃取三次, 合并的有机相, 经无水硫酸钠干燥, 过 滤, 减压浓缩. 得到的粗产品经硅胶柱层析(乙酸乙酯/ 正己烷, 1：10)分离纯化, 得棕色油状液体 2-甲基-8-氨 基喹啉(2) ${ }^{[3]}$ : $628 \mathrm{mg}$, 收率 88\%. ${ }^{1} \mathrm{H}$ NMR $(400 \mathrm{MHz}$, $\left.\mathrm{CDCl}_{3}\right) \delta: 7.93(\mathrm{~d}, J=8.4 \mathrm{~Hz}, 1 \mathrm{H}), 7.25 \sim 7.21(\mathrm{~m}, 2 \mathrm{H})$, $7.10(\mathrm{~d}, J=8.0 \mathrm{~Hz}, 1 \mathrm{H}), 6.89$ (d, $J=8.4 \mathrm{~Hz}, 1 \mathrm{H}), 4.85$ (brs, $2 \mathrm{H}), 2.70(\mathrm{~s}, 3 \mathrm{H})$.

将化合物 $2(2.5 \mathrm{~g}, 15.8 \mathrm{mmol})$ 溶解于二氯甲烷溶液 $(25 \mathrm{~mL})$ 中, 在 $0^{\circ} \mathrm{C}$ 下, 逐滴分别加入乙酰氯 $(4.5 \mathrm{~mL})$ 和 $N, N$-二异丙基乙胺 $(25 \mathrm{~mL})$. 反应体系在 $0{ }^{\circ} \mathrm{C}$ 下搅拌 15 $\min$ 后, 升温至常温并反应过夜. 反应结束后, 减压浓 缩除去溶剂. 粗产物经硅胶柱层析 (乙酸乙酯/正己烷, $V: V=1: 20$ )分离纯化得 $3.15 \mathrm{~g}$ 淡黄色固体 2-甲基8-( $N$-乙酰基)氨基喹啉(3), 收率 99\%. m.p. $69 \sim 71{ }^{\circ} \mathrm{C}$; ${ }^{1} \mathrm{H}$ NMR $\left(400 \mathrm{MHz}, \mathrm{CDCl}_{3}\right) \delta: 9.83(\mathrm{~s}, 1 \mathrm{H}), 8.72(\mathrm{~d}, J=$ $8.8 \mathrm{~Hz}, 1 \mathrm{H}), 8.02(\mathrm{~d}, J=8.4 \mathrm{~Hz}, 1 \mathrm{H}), 7.45 \sim 7.44(\mathrm{~m}, 2 \mathrm{H})$, 7.31 (d, $J=8.4 \mathrm{~Hz}, 1 \mathrm{H}), 2.74$ (s, $3 \mathrm{H}), 2.35$ (s, 3H).

向化合物 3 (3.00 g, $15 \mathrm{mmol})$ 的二氧六环溶液 $(20$ $\mathrm{mL})$ 中, 加入氧化硒 $(5 \mathrm{~g}, 45 \mathrm{mmol})$, 反应在 $85{ }^{\circ} \mathrm{C}$ 下搅 拌 $4 \mathrm{~h}$, 反应体系用硅藻土过滤并用二氯甲烷洗涤滤饼.
合并有机相并减压浓缩.粗产物经硅胶柱层析(乙酸乙 酯/正己烷, $V: V=1: 20)$ 分离纯化, 得 $2.11 \mathrm{~g}$ 淡黄色固 体 2-醛基-8-( $N$-乙酰基)氨基喹啉(4), 收率 65\%. m.p. $105 \sim 107{ }^{\circ} \mathrm{C} ;{ }^{1} \mathrm{H}$ NMR $\left(400 \mathrm{MHz}, \mathrm{CDCl}_{3}\right) \delta: 10.25$ (s, $1 \mathrm{H}), 9.75$ (s, $1 \mathrm{H}), 8.87$ (d, $J=7.6 \mathrm{~Hz}, 1 \mathrm{H}), 8.33$ (d, $J=8.4$ $\mathrm{Hz}, 1 \mathrm{H}), 8.08(\mathrm{~d}, J=8.4 \mathrm{~Hz}, 1 \mathrm{H}), 7.68(\mathrm{~d}, J=8.0 \mathrm{~Hz}, 1 \mathrm{H})$, $7.58(\mathrm{~d}, J=8.4 \mathrm{~Hz}, 1 \mathrm{H}), 2.42(\mathrm{~s}, 3 \mathrm{H})$.

在氩气保护下，向化合物 $4(2.00 \mathrm{~g}, 9.34 \mathrm{mmol})$ 的 1,2 -二氯乙烷溶液 $(30 \mathrm{~mL})$ 中加入 $N, N$-二甲基乙二胺 (3.29 g, $4.08 \mathrm{~mL}, 37.34 \mathrm{mmol})$, 反应混合物在室温下摚 拌 $1 \mathrm{~h}$, 向反应体系中加入 $\mathrm{NaBH}(\mathrm{OAc})_{3}(7.92 \mathrm{~g}, 37.34$ $\mathrm{mmol}$ ), 继续反应 $12 \mathrm{~h}$. 向反应体系中分别加入 $100 \mathrm{~mL}$ 二氯甲烷和饱和 $\mathrm{NaHCO}_{3}$ 水溶液 $(40 \mathrm{~mL})$, 分离有机相 后, 向水相中加入 $2 \mathrm{~mL}$ 氨水, 再用二氯甲烷萃取 3 次. 合并后的有机相减压浓缩. 粗产物溶于 $5 \mathrm{~mL}$ 二氯甲烷, 加入 $6 \mathrm{~mol} \cdot \mathrm{L}^{-1} \mathrm{HCl}(2 \mathrm{~mL})$, 在 $50{ }^{\circ} \mathrm{C}$ 下搅拌反应 $4 \mathrm{~h}$. 向 反应体系中加入 $50 \mathrm{~mL}$ 水并用氨水中和至碱性. 二氯甲 烷萃取 3 次. 合并后的有机相经无水硫酸钠干燥、过滤、 减压浓缩. 粗产物经硅胶柱层析(乙酸乙酯/异丙醇/ $25 \%$ 氨水, $V: V: V=5: 1: 0.2$ )分离纯化得 $683 \mathrm{mg}$ 淡黄色 油状物 2-( $N, N$-二甲基乙二胺基)甲基-8-氨基喹啉(5), 收 率 93\%. ${ }^{1} \mathrm{H}$ NMR $\left(400 \mathrm{MHz}, \mathrm{CDCl}_{3}\right) \delta: 8.01(\mathrm{~d}, J=8.4$ $\mathrm{Hz}, 1 \mathrm{H}), 7.37$ (d, $J=8.4 \mathrm{~Hz}, 1 \mathrm{H}), 7.29(\mathrm{~d}, J=8.0 \mathrm{~Hz}, 1 \mathrm{H})$, $7.12(\mathrm{~d}, J=8.4 \mathrm{~Hz}, 1 \mathrm{H}), 6.91(\mathrm{~d}, J=8.4 \mathrm{~Hz}, 1 \mathrm{H}), 4.97$ (brs, $2 \mathrm{H}), 4.09$ (s, 2H), 2.78 (t, $J=6.0 \mathrm{~Hz}, 2 \mathrm{H}), 2.49$ (t, $J=6.0$ $\mathrm{Hz}, 2 \mathrm{H}), 2.23$ (s, 6H).

向化合物 5 的乙醚溶液中加入过量的盐酸-乙醚溶 液 ( $2.0 \mathrm{~mol} \cdot \mathrm{L}^{-1}$ 盐酸溶解于乙醚中), 将所得沉淀过滤, 乙醚洗涤三次, 减压浓缩得到黄色固体 TDMQ4. m.p. $245{ }^{\circ} \mathrm{C}$ (dec.); ${ }^{1} \mathrm{H}$ NMR $\left(400 \mathrm{MHz}, \mathrm{D}_{2} \mathrm{O}\right) \delta: 8.38$ (d, $J=$ $8.8 \mathrm{~Hz}, 1 \mathrm{H}), 7.95(\mathrm{~d}, J=8.4 \mathrm{~Hz}, 1 \mathrm{H}), 7.81(\mathrm{~d}, J=7.2 \mathrm{~Hz}$, $1 \mathrm{H}), 7.61(\mathrm{t}, J=8.0 \mathrm{~Hz}, 1 \mathrm{H}), 7.57(\mathrm{~d}, J=8.4 \mathrm{~Hz}, 1 \mathrm{H}), 4.76$ (s, 2H), 3.78 3.71 (m, 4H), 2.99 (s, 6H); ${ }^{13} \mathrm{C}$ NMR $(100$ $\left.\mathrm{MHz}, \mathrm{D}_{2} \mathrm{O}\right) \delta: 152.1,139.7,138.7,129.0,128.1,127.0$, 126.7, 124.0, 121.0, 52.6, 51.43, 43.5, 41.9; IR (KBr) v: 3470, 3024, 2798, 2542, 1630, 1601, 1468, 1428, 1397, 1295, 1101, 1084, 1021, 997, 960, 844, 820, 781, 757, 644, $483 \mathrm{~cm}^{-1}$. HRMS (EI) calcd for $\mathrm{C}_{14} \mathrm{H}_{20} \mathrm{~N}_{4}$ : 244.1688, found 244.1684. Anal. calcd for $\mathrm{C}_{14} \mathrm{H}_{20} \mathrm{~N}_{4} \cdot 2.9 \mathrm{HCl} \bullet 0.8 \mathrm{H}_{2} \mathrm{O} \cdot$ $0.03 \mathrm{CH}_{3} \mathrm{COOC}_{2} \mathrm{H}_{5}: \mathrm{C} 46.20, \mathrm{H} 6.79, \mathrm{~N} 15.26$; found $\mathrm{C}$ 46.14, H 6.81, N 15.24.

\section{2 .2 化合物 TDMQ29 的合成}

将 2-甲基-8-硝基喹啉(200 mg, $1.06 \mathrm{mmol}$ )溶解于 DMA (2 mL)中, 分别加入 $\mathrm{FeCl}_{3}(5.2 \mathrm{mg}, 0.032 \mathrm{mmol}$ )和 $\mathrm{K}_{2} \mathrm{~S}_{2} \mathrm{O}_{8}(573 \mathrm{mg}, 2.12 \mathrm{mmol})$. 该反应体系在 $110{ }^{\circ} \mathrm{C}$ 搅拌 
反应 $30 \mathrm{~min}$. 反应结束后, 加水稀释, 用乙酸乙酯萃取 三遍, 合并有机相, 无水硫酸钠干燥, 过滤, 减压浓缩. 得到的粗产品经硅胶柱层析(二氯甲烷/正己烷, $V: V=$ 1:4)分离纯化, 得到 $42 \mathrm{mg}$ 白色固体 2-乙烯基-8-硝基 喹啉(6) ${ }^{[3]}$, 收率 51\%. ${ }^{1} \mathrm{H}$ NMR (400 MHz, $\left.\mathrm{CDCl}_{3}\right) \delta: 8.17$ (d, $J=8.8 \mathrm{~Hz}, 1 \mathrm{H}), 7.97 \sim 7.94(\mathrm{~m}, 2 \mathrm{H}), 7.64(\mathrm{~d}, J=8.4$ $\mathrm{Hz}, 1 \mathrm{H}), 7.52(\mathrm{t}, J=7.6 \mathrm{~Hz}, 1 \mathrm{H}), 7.02 \sim 6.95(\mathrm{~m}, 1 \mathrm{H}), 6.42$ (d, $J=17.6 \mathrm{~Hz}, 1 \mathrm{H}), 5.71(\mathrm{~d}, J=10.8 \mathrm{~Hz}, 1 \mathrm{H}),{ }^{13} \mathrm{C}$ NMR $\left(100 \mathrm{MHz}, \mathrm{CDCl}_{3}\right) \delta: 158.0,148.3,139.3,137.0,136.3$, 131.5, 128.2, 124.7, 123.6, 122.2, 120.6 .

将 2-乙烯基-8-硝基喹啉(6) (100 mg, $0.5 \mathrm{mmol}$ )溶解 于 $2 \mathrm{~mL}$ 1,4-二氧六环中, 分别加入 $\mathrm{Cs}_{2} \mathrm{CO}_{3}(325 \mathrm{mg}, 1.0$ $\mathrm{mmol}$ )、18-冠醚-6 (132 mg, $0.5 \mathrm{mmol}) 、 \mathrm{~N}, \mathrm{~N}$-二甲基-1,2乙二胺 $(109 \mu \mathrm{L}, 1.0 \mathrm{mmol})$. 该反应体系在室温下搅拌反 应 $14 \mathrm{~h}$. 反应结束后, 加水稀释, 用乙酸乙酯萃取三遍, 合并有机相, 无水硫酸钠干燥, 过滤, 减压浓缩. 得到 的粗产品经硅胶柱层析 (乙酸乙酯/异丙醇/氨水 $(25 \%$ ), $V: V: V=5: 1: 0.3)$ 分离纯化, 得到 $66 \mathrm{mg}$ 浅黄色液 体 2-[2'-( $N, N$-二甲基乙二胺基)]乙基-8-硝基喹啉(7a), 收 率 46\%. m.p. 81 83 ${ }^{\circ} \mathrm{C} ;{ }^{1} \mathrm{H}$ NMR $\left(400 \mathrm{MHz}, \mathrm{CDCl}_{3}\right) \delta$ : $8.13(\mathrm{~d}, J=8.4 \mathrm{~Hz}, 1 \mathrm{H}), 7.97(\mathrm{~d}, J=7.6 \mathrm{~Hz}, 2 \mathrm{H}), 7.53$ (t, $J=7.6 \mathrm{~Hz}, 1 \mathrm{H}), 7.42(\mathrm{~d}, J=8.8 \mathrm{~Hz}, 1 \mathrm{H}), 3.24 \sim 3.22(\mathrm{~m}$, $2 \mathrm{H}), 3.16 \sim 3.13(\mathrm{~m}, 2 \mathrm{H}), 2.77(\mathrm{t}, J=6.4 \mathrm{~Hz}, 3 \mathrm{H}), 2.45(\mathrm{t}$, $J=6.4 \mathrm{~Hz}, 2 \mathrm{H}), 2.22(\mathrm{~s}, 6 \mathrm{H}) ;{ }^{13} \mathrm{C} \mathrm{NMR}\left(100 \mathrm{MHz}, \mathrm{CDCl}_{3}\right)$ $\delta: 163.7,147.9,139.0,136.0,131.7,127.6,124.4,123.6$, 123.5, 59.1, 48.0, 47.3, 45.5, 38.4. HRMS (ESI) calcd for $\mathrm{C}_{15} \mathrm{H}_{21} \mathrm{~N}_{4} \mathrm{O}_{2}: 289.1665\left([\mathrm{M}+\mathrm{H}]^{+}\right)$, found 289.1663 .

将化合物 7a (100 mg, $0.35 \mathrm{mmol})$ 溶解于 $3 \mathrm{~mL}$ 乙醇 中, 分别加入二水合氯化亚锡 $(297 \mathrm{mg}, 1.3 \mathrm{mmol}$ )和浓盐 酸 $\left(12 \mathrm{~mol} \cdot \mathrm{L}^{-1}, 1 \mathrm{~mL}\right.$ ). 该反应在 $50{ }^{\circ} \mathrm{C}$ 下搅拌反应 $3 \mathrm{~h}$. 反应结束后, 加冰水淬灭, 向体系中缓慢滴加 $25 \%$ 氨水 中和, 然后用乙酸乙酯萃取三遍. 合并有机相, 无水硫 酸钠干燥, 过滤, 减压浓缩. 得到的粗产品经硅胶柱层 析(乙酸乙酯/异丙醇/氨水 $(25 \%), V: V: V=5: 1: 0.3$ ) 分离纯化, 得 $55 \mathrm{mg}$ 浅黄色液体 2-[2'-( $N, N$-二甲基乙二 胺基)]乙基-8-氨基喹啉(8a), 收率 61\%. ${ }^{1} \mathrm{H}$ NMR (400 $\left.\mathrm{MHz}, \mathrm{CDCl}_{3}\right) \delta: 7.96(\mathrm{~d}, J=8.4 \mathrm{~Hz}, 1 \mathrm{H}), 7.28 \sim 7.23(\mathrm{~m}$, $2 \mathrm{H}), 7.10(\mathrm{~d}, J=7.6 \mathrm{~Hz}, 1 \mathrm{H}), 6.89$ (d, $J=7.6 \mathrm{~Hz}, 1 \mathrm{H}), 5.02$ (brs, $2 \mathrm{H}), 3.17 \sim 3.12(\mathrm{~m}, 4 \mathrm{H}), 2.76(\mathrm{t}, J=6.0 \mathrm{~Hz}, 2 \mathrm{H})$, 2.43 (t, $J=6.4 \mathrm{~Hz}, 3 \mathrm{H}), 2.20(\mathrm{~s}, 6 \mathrm{H}) ;{ }^{13} \mathrm{C}$ NMR $(100 \mathrm{MHz}$, $\left.\mathrm{CDCl}_{3}\right) \delta: 157.9,143.6,137.8,136.3,127.2,126.5,121.9$, $115.8,110.1,59.2,49.0,47.3,45.6,38.5$. HRMS (ESI) calcd for $\mathrm{C}_{15} \mathrm{H}_{23} \mathrm{~N}_{4}[\mathrm{M}+\mathrm{H}]^{+}: 259.1923$, found 259.1925.

将化合物 8a (80 mg, $0.3 \mathrm{mmol}$ ) 溶于乙醚中, 加入过 量的盐酸-乙醚溶液 $(2 \mathrm{~mol} / \mathrm{L})$, 沉淀经过滤, 乙醚洗涤,
减压浓缩得到黄色固体 TDMQ29. m.p. $214 \sim 216{ }^{\circ} \mathrm{C} ;{ }^{1} \mathrm{H}$ NMR (400 MHz, $\left.\mathrm{D}_{2} \mathrm{O}\right) \delta: 8.32(\mathrm{~d}, J=8.8 \mathrm{~Hz}, 1 \mathrm{H}), 7.93(\mathrm{~d}$, $J=8.4 \mathrm{~Hz}, 1 \mathrm{H}), 7.77(\mathrm{~d}, J=8.4 \mathrm{~Hz}, 1 \mathrm{H}), 7.60 \sim 7.52(\mathrm{~m}$, $2 \mathrm{H}), 3.77(\mathrm{t}, J=6.8 \mathrm{~Hz}, 2 \mathrm{H}), 3.61 \sim 3.57(\mathrm{~m}, 4 \mathrm{H}), 3.48(\mathrm{t}$, $J=6.8 \mathrm{~Hz}, 2 \mathrm{H}), 2.94(\mathrm{~s}, 6 \mathrm{H}) ;{ }^{13} \mathrm{C}$ NMR $\left(100 \mathrm{MHz}, \mathrm{CDCl}_{3}\right)$ $\delta: 158.0,139.8,138.1,128.6,127.6,126.6,126.2,123.4$, 123.3, 52.3, 46.3, 43.4, 41.9, 33.2; IR (KBr) v: 3378, 3164, 2828, 2637, 1632, 1599, 1571, 1539, 1504, 1478, 1403, 1322, 1187, 1143, 991, 889, 831, $775 \mathrm{~cm}^{-1}$. HRMS (ESI) calcd for $\mathrm{C}_{15} \mathrm{H}_{23} \mathrm{~N}_{4}[\mathrm{M}+\mathrm{H}]^{+}: 259.1923$, found 259.1913 . Anal. calcd for $\mathrm{C}_{15} \mathrm{H}_{22} \mathrm{~N}_{4} \cdot 3 \mathrm{HCl} \bullet 1.3 \mathrm{H}_{2} \mathrm{O} \bullet 0.05 \mathrm{CH}_{3} \mathrm{COOC}_{2} \mathrm{H}_{5}$ C 46.15, H 7.13, N 14.16; found C 46.15, H 7.16, N 14.18.

\subsection{3 化合物 TDMQ30 的合成}

$N^{1}, N^{1}$-二甲基- $N^{3}$-[2-(2-乙基-8-硝基喹啉)乙基]丙 烷-1,3-二胺(7b) 合成步骤与 7a 相同, 收率 89\%. ${ }^{1} \mathrm{H}$ NMR $\left(400 \mathrm{MHz}, \mathrm{CDCl}_{3}\right) \delta: 8.01(\mathrm{~d}, J=8.4 \mathrm{~Hz}, 1 \mathrm{H}), 7.85(\mathrm{~d}, J=$ $7.6 \mathrm{~Hz}, 2 \mathrm{H}), 7.41$ (t, $J=7.6 \mathrm{~Hz}, 1 \mathrm{H}), 7.30$ (d, $J=8.8 \mathrm{~Hz}$, $1 \mathrm{H}), 3.09$ (t, $J=6.4 \mathrm{~Hz}, 2 \mathrm{H}), 3.00$ (t, $J=6.4 \mathrm{~Hz}, 2 \mathrm{H}), 2.93$ (brs, $1 \mathrm{H}), 2.61(\mathrm{t}, J=7.2 \mathrm{~Hz}, 2 \mathrm{H}), 2.22(\mathrm{t}, J=7.2 \mathrm{~Hz}, 2 \mathrm{H})$, $2.20(\mathrm{~s}, 6 \mathrm{H}), 1.65 \sim 1.58(\mathrm{~m}, 2 \mathrm{H}) ;{ }^{13} \mathrm{C}$ NMR $(100 \mathrm{MHz}$, $\left.\mathrm{CDCl}_{3}\right) \delta: 163.8,147.8,138.8,135.9,131.6,127.5,124.4$, 123.6, 123.4, 57.9, 48.3, 47.9, 45.4, 38.1, 27.9. HRMS (ESI) calcd for $\mathrm{C}_{16} \mathrm{H}_{23} \mathrm{~N}_{4} \mathrm{O}_{2}[\mathrm{M}+\mathrm{H}]^{+}: 303.1821$, found 303.1823.

$N^{1}, N^{1}$-二甲基- $N^{3}$-(2-(2-乙基-8-氨基喹啉)乙基)丙 烷-1,3-二胺 $(8 b)$ 合成步骤与 8a 相同, 收率 73\%. ${ }^{1} \mathrm{H}$ NMR $\left(400 \mathrm{MHz}, \mathrm{CDCl}_{3}\right) \delta: 7.94(\mathrm{~d}, J=8.4 \mathrm{~Hz}, 1 \mathrm{H}), 7.29 \sim 7.22$ (m, 2H), $7.09(\mathrm{~d}, J=8.0 \mathrm{~Hz}, 1 \mathrm{H}), 6.88(\mathrm{~d}, J=7.2 \mathrm{~Hz}, 1 \mathrm{H})$, 5.00 (brs, $2 \mathrm{H}$ ), $3.13 \sim 3.11$ (m, 4H), 2.69 (t, $J=7.2 \mathrm{~Hz}$, $2 \mathrm{H}), 2.27$ (t, $J=7.2 \mathrm{~Hz}, 2 \mathrm{H}), 2.16(\mathrm{~s}, 6 \mathrm{H}), 1.68 \sim 1.61(\mathrm{~m}$, $2 \mathrm{H}) ;{ }^{13} \mathrm{C}$ NMR $\left(100 \mathrm{MHz}, \mathrm{CDCl}_{3}\right) \delta: 157.8,143.6,137.8$, 136.3, 127.2, 126.6, 121.9, 115.8, 110.0, 58.0, 49.1, 48.3, 45.5, 38.6, 28.0. HRMS (ESI) calcd for $\mathrm{C}_{16} \mathrm{H}_{25} \mathrm{~N}_{4}$ : $273.2079\left([\mathrm{M}+\mathrm{H}]^{+}\right)$, found 273.2083 .

TDMQ30 合成步骤与 TDMQ29 相同, m.p. 220 $222{ }^{\circ} \mathrm{C} ;{ }^{1} \mathrm{H}$ NMR $\left(400 \mathrm{MHz}, \mathrm{D}_{2} \mathrm{O}\right) \delta: 8.36(\mathrm{~d}, J=8.4 \mathrm{~Hz}$, 1H), 7.97 (d, $J=8.4 \mathrm{~Hz}, 1 \mathrm{H}), 7.78(\mathrm{~d}, J=7.2 \mathrm{~Hz}, 1 \mathrm{H})$, $7.63 \sim 7.55(\mathrm{~m}, 2 \mathrm{H}), 3.70(\mathrm{t}, J=7.2 \mathrm{~Hz}, 2 \mathrm{H}), 3.46(\mathrm{t}, J=$ $7.2 \mathrm{~Hz}, 2 \mathrm{H}), 3.23 \sim 3.19(\mathrm{~m}, 4 \mathrm{H}), 2.86(\mathrm{~s}, 6 \mathrm{H}), 2.20 \sim 2.11$ (m, 2H); ${ }^{13} \mathrm{C}$ NMR (100 MHz, $\left.\mathrm{CDCl}_{3}\right) \delta: 158.2,139.5$, 138.2, 128.5, 127.5, 126.7, 126.2, 123.4, 123.3, 54.3, 45.7, 44.5, 42.9, 33.2, 21.2; IR (KBr) v: 3429, 2956, 2483, 1632, 1599, 1468, 1421, 1391, 1322, 1278, 1185, 1081, 998, 968, 840, 781, 647, 570, $474 \mathrm{~cm}^{-1}$. HRMS (EI) calcd for $\mathrm{C}_{16} \mathrm{H}_{24} \mathrm{~N}_{4}:$ 272.2001, found 272.1995. Anal. calcd for 
$\mathrm{C}_{16} \mathrm{H}_{24} \mathrm{~N}_{4} \cdot 2.7 \mathrm{HCl} \cdot 1.6 \mathrm{H}_{2} \mathrm{O} \bullet 0.04 \mathrm{CH}_{3} \mathrm{COOC}_{2} \mathrm{H}_{5}: \mathrm{C} 48.14, \mathrm{H}$ 7.55, N 13.90; found C 48.15, H 7.24, N 13.89 .

3.2 .4 紫外一可见光谱法测定化合物与铜、锌离子的 络合常数

参照文献方法 ${ }^{[3,5,20]}$, 采用紫外-可见光谱法测定化 合物与铜、锌离子的络合常数. 具体实验方法及步骤如 下

母液的配制: 螯合剂(L)配成 $3 \mathrm{mmol} \cdot \mathrm{L}^{-1}$ 的水溶液, 竞争剂 $(\mathrm{C}=\mathrm{EDTA}, \mathrm{EGTA}, \mathrm{NTA}, \mathrm{EDA})$ 配成 $3 \mathrm{mmol} \cdot \mathrm{L}^{-1}$ 水溶液, $\mathrm{CuSO}_{4}$ 和 $\mathrm{ZnSO}_{4}$ 配成 $15 \mathrm{mmol} \cdot \mathrm{L}^{-1}$ 的水溶液, 实 验在 Tris $\cdot \mathrm{HCl}\left(20 \mathrm{mmol} \cdot \mathrm{L}^{-1}\right) / \mathrm{NaCl}\left(150 \mathrm{mmol} \cdot \mathrm{L}^{-1}\right)$ 缓冲 溶液( $\mathrm{pH}$ 7.4) 中进行.

实验步骤: 在 $5 \mathrm{~mL}$ 离心管中加入 $20 \mu \mathrm{L}$ 螯合剂(L), $20 \mu \mathrm{L} \mathrm{CuSO}_{4}\left(3 \mathrm{mmol} \cdot \mathrm{L}^{-1}\right)$ 或 $20 \mu \mathrm{L} \mathrm{ZnSO}_{4}\left(3 \mathrm{mmol} \cdot \mathrm{L}^{-1}\right)$, $20 \mu \mathrm{L}$ 竞争剂(EDTA 或 EGTA 或 NTA 或 EDA, 3 mmol• $\mathrm{L}^{-1}$ ), 最后加入 $20 \mathrm{mmol} \cdot \mathrm{L}^{-1} \mathrm{Tris} \cdot \mathrm{HCl}$ 缓冲溶液至 $4 \mathrm{~mL}$. 化合物 $\mathbf{L}$, 竞争剂(EDTA 或 EGTA 或 NTA 或 EDA)和 $\mathrm{Cu}^{2+}$ 最终浓度分别为 $15 \mu \mathrm{mol} \cdot \mathrm{L}^{-1}$. 待螯合剂与金属离 子相互作用达到平衡后，反应体系中的螯合剂浓度(L)、 金属离子浓度(M)、金属-螯合剂复合物(L-M)的浓度与 各组分的紫外吸收变化相关, 通过紫外-可见光谱仪测 试得到. 紫外-可见光谱仪进行全谱扫描, 记录 200 $500 \mathrm{~nm}$ 的吸收谱图, 继而采用Beer-Lambert 比耳定律计 算出螯合剂对不同金属离子的亲合常数. 选择竞争剂 (C), 竞争剂一铜离子络合物(C-M)无明显吸收的波长下 计算.

螯合剂与铜离子的络合常数为:

$\log K_{\text {app(L-Cu })}=\log K_{\text {app(C-Cu })}+2 \log (x /(1-x))$

其中, $x$ 表示铜离子与化合物络合的百分比, $x=\left(A_{\mathrm{L}}-\right.$ $\left.A_{\mathrm{L}-\mathrm{Cu}-\mathrm{C}}\right) /\left(A_{\mathrm{L}}-A_{\mathrm{Cu}-\mathrm{L}}\right)$.

3.2 .5 紫外滴定法测化合物与铜离子、锌离子的络合 计量比

参照文献方法 ${ }^{[3,5,20 ~ 22]}$, 测定化合物与铜、锌离子的 络合计量比. 具体实验方法及步骤如下:

母液的配制: 螯合剂(L)配成 $3 \mathrm{mmol} \cdot \mathrm{L}^{-1}$ 的水溶液, $\mathrm{CuSO}_{4}$ 和 $\mathrm{ZnSO}_{4}$ 配成 $15 \mathrm{mmol} \cdot \mathrm{L}^{-1}$ 的水溶液, 实验在 Tris $\bullet \mathrm{HCl}\left(20 \mathrm{mmol} \cdot \mathrm{L}^{-1}\right) / \mathrm{NaCl}\left(150 \mathrm{mmol} \cdot \mathrm{L}^{-1}\right)$ 缓冲溶液 (pH 7.4)中进行.

实验步骤: 在 $5 \mathrm{~mL}$ 离心管中加入 $20 \mu \mathrm{L}$ 化合物 $\mathbf{L}$ (1.0 当量), 加入不同 equiv. 的 $\mathrm{CuSO}_{4}$ 或 $\mathrm{ZnSO}_{4}(0.2 \sim 10$ 当量), 最后加入 $\mathrm{Tris} \cdot \mathrm{HCl}$ 缓冲溶液, 使最终的溶液体积 为 $4 \mathrm{~mL}$. 用紫外分光光度计进行全谱扫描，记录 200 $500 \mathrm{~nm}$ 的谱图. 在某一波长下(铜离子、锌离子在此处
应无明显吸收)作出物质的量与吸光度的关系图.

辅助材料(Supporting Information) 化合物 TDMQ4, TDMQ29, TDMQ30 的 ${ }^{1} \mathrm{H}$ NMR, ${ }^{13} \mathrm{C}$ NMR, HRMS，以及 化合物与 $\mathrm{Cu}(\mathrm{II})$ 和 $\mathrm{Zn}(\mathrm{II})$ 的化学计量比、络合常数的谱 图. 这些材料可以免费从本刊网站(http://sioc-journal. $\mathrm{cn} /)$ 上下载.

\section{References}

[1] Telpoukhovskaia, M. A.; Orvig, C. Chem. Soc. Rev. 2013, 42, 1836.

[2] Gaggelli, E.; Kozlowski, H.; Valensin, D.; Valensin, G. Chem. Rev. 2006, 106, 1995.

[3] Zhang, W.; Huang, D.; Huang, M.; Huang, J.; Wang, D.; Liu, X.; Nguyen, M.; Vendier, L.; Mazères, S.; Robert, A.; Liu, Y.; Meunier, B. ChemMedChem. 2018, 13, 684 .

[4] Robert, A.; Liu, Y.; Nguyen, M.; Meunie, B. Acc. Chem. Res. 2015 48(5), 1332

[5] Nguyen, M.; Rechignat, L.; Robert, A.; Meunier, B. ChemistryOpen 2015, 4, 27.

[6] Bolea, I.; Gella, A.; Unzeta, M. J. Neural Transm. 2013, 120, 893.

[7] Cummings, J. L.; Travis, M.; Zhong, K. Alzheimer's Res. Ther. 2014, 6, 37.

[8] Scarpini, E.; Scheltens, P.; Feldman, H. Lancet Neurol. 2003, 2, 539.

[9] Nguyen, M.; Huang, M.; Liu, Y.; Meunier, B.; Robert, A. C. $R$ Chim. 2017, 20, 987

[10] Viles, J. H. Coord. Chem. Rev. 2012, 256, 2271.

[11] Arena, G.; Pappalardo, G.; Sovago, I.; Rizzarelli, E. Coord. Chem. Rev. 2012, 256, 3.

[12] Migliorini, C.; Porciatti, E.; Luczkowski, M.; Valensin, D. Coord. Chem. Rev. 2012, 256, 352

[13] Drew, S. C.; Barnham, K. J. Acc. Chem. Res. 2011, 44, 1146.

[14] Crapper, D. R.; Dalton, A. J.; Kruck, T. P.; Bell, M. Y.; Smith, W. L.; Kalow, W.; Andrews, D. F. Lancet 2011, 337, 1304.

[15] Ritchie, C. W.; Bush, A. I.; Mackinnon, A.; Macfarlane, S.; Mastwyk, M.; MacGregor, L.; Kiers, L.; Cherny, R.; Li, Q. X.; Tammer, A.; Carrington, D.; Mavros, C.; Volitakis, I.; Xilinas, M.; Ames, D.; Davis, S.; Volitakis, I.; Xilinas, M.; Ames, D.; Davis, S.; Beyreuther, K.; Tanzi, R. E.; Masters, C. L. Arch. Neurol. (Chicago) 2003, 60, 1685.

[16] Adlard, P. A.; Cherny, R. A.; Finkelstein, D. I.; Gautier, E.; Robb, E.; Cortes, M.; Volitakis, I.; Liu, X.; Smith, J. P.; Perez, K.; Laughton, K.; Li, Q. X.; Charman, S. A.; Nicolazzo, J. A.; Wilkins, S.; Deleva, K.; Lynch, T.; Kok, G.; Ritchie, C. W.; Tanzi, R. E.; Cappai, R.; Masters, C. L.; Barnham, K. J.; Bush, A. I. Neuron 2008, 59,43

[17] Bareggi, S. R.; Cornelli, U. CNS Neurosci. Ther. 2012, 18, 41

[18] Cherny, R. A.; Atwood. C. S.; Xilinas, M. E.; Gray, D. N.; Jones, W. D.; McLean, C. A.; Barnham, K. J.; Volitakis, I.; Fraser, F. W.; Kim, Y.; Huang, X.; Goldstein, L. E.; Moir, R. D.; Lim, J. T.; Beyreuther, K.; Zheng, H.; Tanzi, R. E.; Masters, C. L.; Bush, A. I. Neuron 2001, 30, 665 .

[19] Ceccom, J.; Cosledan, F.; Halley, H.; Frances, B.; Lassalle, J. M.; Meunier, B. Plos One 2012, 7, e43105.

[20] Ngyyen. M.; Robert, A.; Sournia-Saquet, A.; Vendier, L.; Meunier. B. Chem.-Eur. J. 2014, 20,6771.

[21] Zhang, W.; Liu, Y.; Hureau, C.; Robert, A.; Meunier B. Chem.-Eur. J. 2018, 24, 7825 .

[22] Zhang, W.; Huang, M.; Bijani, C.; Liu, Y.; Robert, A.; Meunier, B. C. R. Chim. 2018, 21, 745 . 